\section{P.1.25 THE EFFECT OF VARIATIONS AIR POLLUTION CONCENTRATION ON ISCHEMIC STROKE}

${ }^{1}$ Gwan-Ling Lin*, ${ }^{2}$ Wei-Te Wu*, ${ }^{2}$ Saou-Hsing Liou, ${ }^{3}$ Trong-Neng Wu. ${ }^{1}$ Nursing, Cardinal Tien Junior College Of Healthcare And Management, New Taipei City, Taiwan, Taipei City, Taiwan; ${ }^{2}$ National Institute of Environmental Health Sciences, National Health Research Institutes, Zhunan, Miaoli, Taiwan; ${ }^{3}$ Department of Healthcare, Asia University, Taichung, Taiwan

\subsection{6/OEM-2019-EPI.227}

Background Stroke is the third most common cause of death in Taiwan. However, the reasonable range in time scale of air pollution concentration for inducing ischemic stroke has not been clear. Therefore, the purpose of the study was to examine the effect of time scale air pollution concentration and stroke risk.

Methods We recruited 256 ischemic stroke patients in emergency department and 98 healthy controls from a Taiwan Medical Center between 2012 and 2013. The subjects were interviewed in person and completed the demographic, disease history questionnaires. Each participant provided blood samples for metal and oxidative stress measurements. We used the geographic information system (GIS) and the Inverse distance weighting (IDW) model to estimate the concentrations of air pollutants (PM2.5, PM10, SO2, NO2, CO, O3) at home address from 75 monitoring stations in Taiwan at different time scales ( 1 day, 2 days, 3 days, 7 days, 14 days, 1 month, 7 months, 1 year) before blood drawing. Logistic regression models were performed to estimate the odds ratio (OR) for stroke risk.

Results The interquartile range (IQR) PM2.5 concentrations were $17.5,16.3,14.5,14.4,12.0,9.2,6.6$, and $1.9 \mu \mathrm{g} / \mathrm{m} 3$ respectively, for different time scales within 1 day, 2 days, 3 days, 7 days, 14 days, 1 month, 7 months, 1 year period before stroke onset. We found that the 7 days, 14 days, 1 month PM2.5 concentrations were significantly increased risk of stroke, after adjustment for smoking, environmental tobacco smoke, ever stroke, family history of stroke, hypertension, hyperlipidemia, type 2 diabetes, heart disease history, temperature, and relative humidity. The results also showed that elevated stroke risks with 2 days, 3 days, 7 days, 14 days, 1 month PM10, and with 7 days, 14 days, and 1 month O3.

\section{P.1.26 BIOMARKERS OF HEALTH EFFECTS IN NANOMATERIALS WORKERS: UPDATED STATUS OF NANOEPIDEMIOLOGY}

Saou-hsing Liou*, Wei-Jin Li, Wei-Te Wu. National Health Research Institutes, Miaoli County, Taiwan

\subsection{6/OEM-2019-EPI.228}

The first article 'Epidemiological Study of Health Hazards among Workers Handling Engineered Nanomaterials.' was published on J Nanopart Res in 2012, and the first review article 'Assessing the first wave of epidemiological studies of nanomaterial workers' was published on J Nanopart Res in 2015. Until now, 29 epidemiological studies were published in peer-reviewed scientific journal. In addition, 5 epidemiological studies were traced but unpublished in the peer-reviewed journal, including $1 \mathrm{PhD}$. thesis and 4 conference abstracts. Most of these studies involved single nanomaterial exposure, for example, 8 articles for carbon nanotubes (MWCNT), 7 articles for titanium dioxide, 1 article for iron oxides, nano calcium carbonate, nano zinc oxide, and nanocomposite, but 6 studies from Taiwan involved multiple exposures. Two studies did not mention the specific components of nanoparticles. Most of these studies were done in Europe (14), followed by Asia (10), USA (2) and Australia (1).

Generally, biomarkers were used as the outcomes variables to elaborate the health hazards of nanomaterials, except for 1 study surveyed work-related symptoms and diseases worsen by work. Exhaled breath condensate (EBC) and serum were the most frequently used biospecimen. All 26 cross-sectional studies and 1 six-month longitudinal panel study showed positive relationship between nanomaterials exposures and various biomarkers. Positive health effects include: 1. elevation of lung fibrosis markers and lung inflammation markers; 2. elevation of cardiovascular injury markers and abnormal HRV; 3. elevation of EBC nucleic acid, lipid and protein oxidative markers; 4. Increased immune markers; 5. changes in the ncRNA and mRNA expression, reduced global methylation, and specific gene methylation.

This review provides some insight into potentially adverse effects that might be related to nanomaterial exposures and provides a foundation for future work. We expect more longitudinal studies with repeated measurements to explore chronic and cumulative effects of nanomaterial exposure.

\section{P.1.28 AIR POLLUTION AND DISEASES: A PRACTICAL TOOL FOR EFFECTIVE PUBMED SEARCH}

Stefania Curti ${ }^{*}$, Stefano Mattioli. University Of Bologna, Bologna, Italy

\subsection{6/OEM-2019-EPI.229}

Objectives To identify efficient PubMed search filters for the study of outdoor air pollution determinants of diseases.

Methods We listed Medical Subject Headings (MeSH) and non-MeSH terms that seemed pertinent to outdoor air pollutants exposure as determinants of diseases. Proportions of potentially pertinent articles retrieved by each term were estimated. We then formulated two filters: one 'more specific', one 'more sensitive'. Their performances were compared with a gold standard of systematic reviews on associations between diseases and outdoor air pollution. We calculated, for both the filters, the number (of abstract) needed to read (NNR) to identify one potentially pertinent article, exploring three diseases potentially associated with outdoor air pollution.

Results The combination of terms that yielded a threshold of potentially pertinent articles $\geq 40 \%$ permitted to formulate the 'more specific' filter. On the basis of the combination of all search terms under study we formulated the 'more sensitive' filter. In comparison with the gold standard, the 'more specific' filter had the highest specificity $(67.4 \%$; with a sensitivity of $82.5 \%$ ) and the 'more sensitive' filter had the highest sensitivity (98.5\%; with a specificity of $47.9 \%)$. For the 'more specific' filter and the 'more sensitive' one the NNR to find one potentially pertinent article was 1.9 and 3.3, respectively.

Conclusions The proposed search filters help investigating environmental determinants of medical conditions. We published them on: Curti $S$ et al. PubMed search filters for the study of putative outdoor air pollution determinants of disease. BMJ Open. 2016;6 (12):e013092. 